\title{
Rats remember not wisely but too well
}

\author{
SARAH ADAMS-PEPPER \\ University of Hawaii, Honolulu, Hawaii \\ SYLVAIN GAGNON \\ Université Laval, Quebec, Quebec, Canada \\ and \\ SUSAN FORE and H. L. ROITBLAT \\ University of Hawaii, Honolulu, Hawaii
}

\begin{abstract}
Rats were trained in a three-alternative spatial delayed matching-to-sample task in a starburst maze. Samples consisted of rewarded forced choices of one arm, and retention was indicated by rats' returning to that arm after a 90-sec delay. If a rat made an error on its first choice, it was returned to the start compartment and allowed a second choice. Unlike in previous experiments with this task, all three arms were available during the animals' second choices. The rats tended to perseverate in their second choices by returning to the arm that they had erroneously visited on their first choice. In Experiment 1, the accuracy of second choices following first-choice errors was below chance during the first block of sessions, when a 90 -sec delay intervened between the first choice and the second choice, and at chance during the second block of sessions, when a short (5-6 sec) delay intervened between first and second choices. In Experiment 2, long-delay and shortdelay sessions were randomly presented to naive subjects. Similar results were obtained. In both experiments, the tendency to repeat the erroneous first choice was greater when long delays separated the two choices than when short delays were used. The results suggest that rats make their first-choice errors because they erroneously encode or remember the location of the sample and that they base their second choices on the same erroneous memory. The increase in perseveration at long delays implies some kind of rehearsal-like mechanism that slows forgetting of the memory controlling the first choice.
\end{abstract}

Several studies have examined animals' abilities to perform a delayed matching-to-sample task in which three alternatives are presented on every trial and second choices are permitted following first-choice errors. Both pigeons (see, e.g., Dale, 1988; Roitblat, 1980; Roitblat \& Scopatz, 1983) and rats (Roitblat \& Harley, 1988; Roitblat, Harley, \& Helweg, 1989) show higher than chance choice accuracy on these second choices, thereby indicating that they have more information about the sample than that reflected in their erroneous first choices. For example, a simple all-or-none encoding model assumes that the animal either has complete information (all) or no information (none) about the sample when making its first choice. According to this model, the occurrence of a firstchoice error must mean that the animal has no information about the identity of the correct choice, and all subsequent choices based on the same information must also be pure guesses. Because they are assumed to be pure guesses, these subsequent choices are predicted to be no more accurate than chance. Above-chance second-choice

This research was supported by National Science Foundation Grant BNS 85-05681. Request reprints from H. L. Roitblat, Department of Psychology, University of Hawaii, 2430 Campus Road, Honolulu, HI 96822 (e-mail: roitblat@uhunix.uhcc.hawaii.edu). accuracy, in contrast, indicates that the animal does have some information about the identity of the sample when it makes its first-choice error, but the expression of that information is somehow prevented.

In most previous experiments done with the secondchoice procedure, the alternative that is erroneously selected on the first choice is removed before the animal is allowed to make its second choice. As a result, the alternative consistent with the most salient, but misleading, information has been removed, and the animal has to select on the basis of less salient alternatives. The cause of the animal's first-choice error is therefore left obscure.

In studies of three-alternative matching in rats, a spatial version of the task has been performed in a starburst maze. Each trial begins with the placement of the rat in a start compartment in the stem of the maze. The stem leads to three goal arms. One goal arm is baited, and the rat is allowed to run into that goal arm (forced choice sample run) to retrieve a piece of food (a piece of Froot Loop, a presweetened breakfast cereal). The rat is then returned to the start compartment and allowed a free choice among all three arms. If the rat returns to the arm in which it has received a sample reward at the start of the trial, it receives another, larger reward, and the trial is over. If it enters one of the other arms, the door to that arm is closed, the rat is returned to the start compartment, and 
a second choice is permitted between the remaining two arms. Correct second choices are also rewarded. Rats learn this task readily, and, as noted above, both their first choices and their second choices following firstchoice errors are more accurate than chance (Roitblat \& Harley, 1988; Roitblat et al., 1989).

Although it is clear that rats have information about the sample even when they make first-choice errors, it is not clear why they make these errors. For some reason, at the time of the rat's first choice, its "memory" for an erroneous arm is more salient than its memory for the correct arm. This difference in salience could be mediated by many different kinds of mechanisms (see Roitblat, 1982, 1987, for reviews), including differences in trace strength (see, e.g., Grant \& Roberts, 1973; Roberts \& Grant, 1976) or inattention to the task during the first choice (see, e.g., Roitblat, 1980, 1984a). One class of model attributes first-choice errors to failures in the encoding or maintenance of the memory for the sample. The animal may fail to properly identify or encode the location of the arm in which it has received its sample reward. Alternatively, it may correctly encode the information, but variability in its memory may modify its representation of the sample arm during the retention interval. A second class of explanation attributes first-choice errors to momentary fluctuations in the animal's memory or to momentary failures to use this information. In previous tasks done with the second-choice procedure, it has not been possible to discriminate between these two classes of models because the erroneous first choices were unavailable when the animals were required to make their second choices. In studies done with rats, for example, subjects have been prevented from reentering the erroneously chosen arm because its entry door is closed. If rats make first-choice errors because of momentary fluctuations in their memories, they should choose accurately when they are allowed a second choice even if the erroneously chosen arm remains available. Momentary fluctuations presumably do not destroy the memory, they merely prevent it temporarily from controlling the animal's choice. Because first-choice performance is generally accurate, these fluctuations, if they occur, must tend to return the animal's memory to the correct state. Therefore, the rat is most likely to base its second choice on its proper representation rather than on the perturbed representation and thereby to make a correct choice. On the other hand, if first-choice errors derive from an encoding failure or from a permanent perturbation in the rat's memory, rats may be expected to choose the arm most consistent with memory, and this choice is then likely to be a reentry of the arm erroneously visited during the first choice.

In the present study, we examined performance when the erroneously chosen first-choice arm remained available during a second choice. Memory loss during the sample-choice interval predicted chance responding during second choices, following first-choice errors. Temporary fluctuations or inattention during the first choice predicted above-chance second-choice accuracy. Encoding failure or permanent change in the memory predicted below-chance second-choice accuracy and perseveration of the erroneous first-choice response.

\section{EXPERIMENT 1}

\section{Method}

Subjects. The subjects were 3 male Sprague-Dawley rats from Simonsen Laboratory in Gilroy, CA. They were kept in individual cages, under a 14:10-h light:dark regime. The rats were fed daily rations of Purina Rat Chow in sufficient quantities to maintain approximately $85 \%$ of their free-feeding weights. They had free access to water at all times, except when on the maze. All subjects were highly experienced in the spatial delayed matching-to-sample task, having served in previous studies (Roitblat \& Harley, 1988; Roitblat et al., 1989). The rats were tested prior to the start of the present experiment, to ensure that their performance was still adequate.

Apparatus. All tests were conducted in the three-arm starburst maze used in the previous studies (Roitblat \& Harley, 1988; Roitblat et al., 1989). Briefly, the maze consisted of a stem that connected a start compartment to three goal arms. The start compartment and the goal arms were separated from the rest of the maze by guillotine doors. The maze was elevated above the floor in a well-lit experimental room containing a window at one end, a door at the other, and other laboratory odds and ends. A food cup was recessed in the floor of the maze near the end of each goal arm. The subjects could not see the contents of this food cup prior to entering the goal arm. A screen made of aluminum window screening edged with masking tape was used to separate a sample reward (1/4 to $1 / 3$ Froot Loop) from the choice reward (a whole Froot Loop, weighing approximately $210 \mathrm{mg}$ ). The choice reward was placed beneath the screen, where it could be seen and smelled but not eaten.

Procedure. The subjects were tested in once-daily sessions of 24 trials each. Each trial consisted of a forced-choice sample run and one or two choice runs. At the start of the trial, one randomly preselected arm was baited with a whole Froot Loop and the bait cup was covered with a screen. An additional piece of Froot Loop was put on top of the screen and the rat was placed in the startbox for the sample run. In the sample run, only the door leading to the baited arm was opened; all other doors were closed. The rat was then released from the start compartment and allowed to run through the maze and into the goal arm to consume the sample reward. The animal was subsequently returned to the start compartment and contained there for a $90-\mathrm{sec}$ interval before being allowed to make its first test choice. The doors to all three arms were then opened, and the screen covering the choice reward was removed. The animal was again released from the start compartment and allowed to choose one of the three arms. If it chose the arm containing the choice reward, it was allowed to consume the Froot Loop and was then returned to its cage for the intertrial interval. Alternatively, if the rat made an incorrect first choice, it was returned to the start compartment and released to make a second choice among the three arms.

Two retention-interval durations between first-choice errors and second choices were tested. On short-delay trials, the rats were released from the start compartment as soon as the doors could be set $(5-6 \mathrm{sec})$. In the long-delay condition, they were returned to their home cages for $90 \mathrm{sec}$ before being returned to the start compartment for the second choice. The short retention intervals were intended for comparing second-choice accuracy with first-choice accuracy during spatial delayed matching-to-sample training. The long delays were intended for comparing second-choice performance with first-choice performance, with a delay comparable to that between the sample and the first choice. Following correct second choices, rats were allowed to eat the choice reward, but if they again 
chose incorrectly, they were returned to their cages unfed for the intertrial interval. Intertrial intervals were nominally $60 \mathrm{sec}$ and began following an erroneous second choice or following consumption of the choice reward. In all cases, the delay between the sample run and the first choice was $90 \mathrm{sec}$. Five long-delay sessions were run followed by five short-delay sessions.

\section{Results}

Figure 1 shows first- and second-choice accuracy on all sessions in Experiment 1. First-choice accuracy in the present experiment was comparable to (or better than) that reported earlier (Roitblat \& Harley, 1988). As would be expected, given that the delay occurred following the first choice, first-choice accuracy was approximately equal in both delay conditions, $75.6 \%$ for short-delay trials and $79.4 \%$ for long-delay trials $[t(4)=0.50, p>.05]$. Second-choice accuracy, however, was not above chance (chance was 33\% in this experiment, compared with $50 \%$ in previous experiments). With short delays between first and second choices, session average second-choice accuracy $(32.9 \%)$ did not differ from chance $[t(2)=-.01$, $p>.05]$. With long delays, however, choice accuracy $(15.7 \%)$ was significantly below chance $[t(2)=-5.67$, $p<.05$ ].

The major determinants for the identity of the animal's second choice were the locations of the sample and the location of its first choice. Table 1 shows the frequency with which each first choice followed each sample. Consistent with the findings of above-chance accuracy, first choices were distributed nonrandomly relative to the sample for both long delays $\left[\chi^{2}(4, n=360)=347.73\right.$,

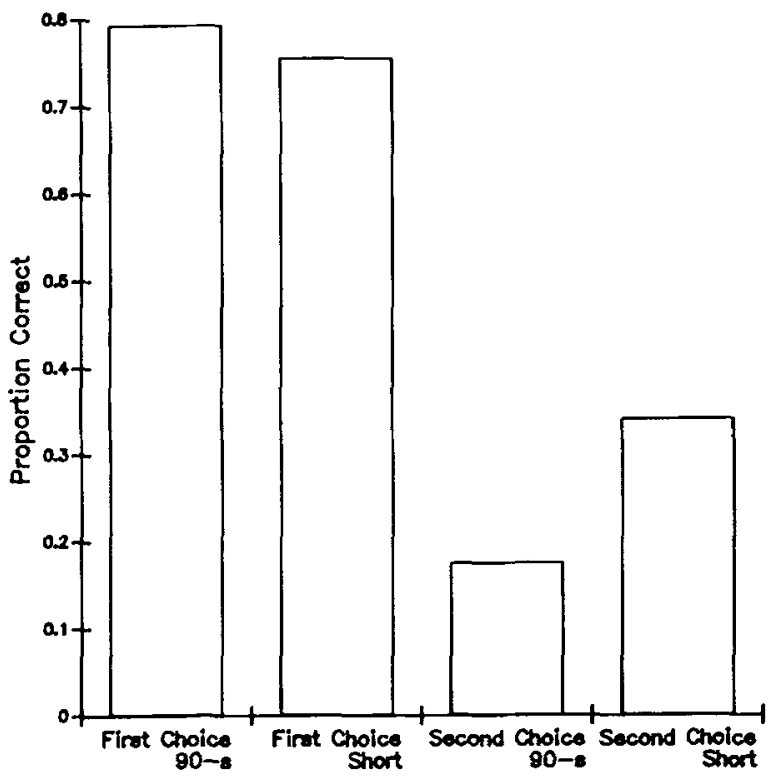

Figure 1. Mean first- and second-choice accuracy throughout Experiment 1 . The first bar shows first-choice accuracy with a 90sec delay between first and second choices. The second bar shows first-choice accuracy with a 5-6-sec delay between choices. The next two bars show second-choice accuracy for short- and long-delay conditions, respectively.
Table 1

First Choices Following Each Sample in Experiment 1

\begin{tabular}{clcr} 
& \multicolumn{3}{c}{ First Choice } \\
\cline { 2 - 4 } Sample & A & B & C \\
\hline & Short Delay & \\
A & 94 & 12 & 14 \\
B & 17 & 87 & 16 \\
C & 20 & 9 & 91 \\
& Long Delay & & \\
A & 100 & 6 & 14 \\
B & 13 & 99 & 8 \\
C & 19 & 14 & 87 \\
\hline
\end{tabular}

Note-Values are the frequencies with which each of the three arms was selected following each sample. A total of 720 trials were presented.

Table 2

Second Choices Following Each Sample in Experiment 1

\begin{tabular}{cccc} 
& \multicolumn{3}{c}{ Second Choice } \\
\cline { 2 - 3 } Sample & A & B & C \\
\hline & Short Delay & & \\
A & 13 & 5 & 8 \\
B & 16 & 7 & 10 \\
C & 16 & 3 & 10 \\
& Long Delay & & \\
A & 5 & 4 & 11 \\
B & 11 & 3 & 7 \\
C & 16 & 12 & 5 \\
\hline
\end{tabular}

Note-Values are the frequencies with which each of the three arms was selected as the second choice following each sample. Second choices were permitted only following first-choice errors. "Short delay" indicates that the interval between the end of the first choice and the start of the second choice was as short as the experimenter could manage (about 5-6 sec). "Long delay" indicates that the interval between the choices was at least $90 \mathrm{sec}$ in duration.

$p<.01]$ and short delays $\left[\chi^{2}(4, n=360)=291.15\right.$, $p<.01]$. The locations of first-choice errors, however, were found to be independent of the sample $\left[\chi^{2}(1\right.$, $n=88)=0.79, p>.05$, and $\chi^{2}(1, n=74)=2.77$, $p>.05$, for short-delay and long-delay conditions, respectively]. ${ }^{1}$

Table 2 shows the frequency with which each second choice followed each sample. Consistent with chance, second choices were found to be distributed randomly relative to the sample on trials with a short delay between first and second choices $\left[\chi^{2}(4, n=88)=1.41, p>.05\right]$ In contrast, when a long delay intervened between first and second choices, the relationship between samples and second choices was nonrandom $\left[\chi^{2}(4, n=74)=11.34\right.$, $p<.05$ ]. Recall that second-choice accuracy was below chance in the long-delay condition and that the distribution of second choices following each sample shows that correct second choices were less frequent than expected under the assumption of independence between samples and second choices.

Table 3 shows the frequency with which each arm was selected as the second choice following the commission of a first-choice error. Under both delay conditions, rats 
Table 3

Second Choices Following Each First Choice in Experiment 1

\begin{tabular}{cccc} 
& \multicolumn{2}{c}{ Second Choice } \\
\cline { 2 - 4 } First Choice & A & B & C \\
\hline \multicolumn{5}{c}{ Short Delay } & & \\
B & 30 & 3 & 4 \\
C & 9 & 6 & 6 \\
& 6 & 6 & 18 \\
A & Long Delay & & \\
B & 27 & 2 & 3 \\
C & 2 & 16 & 2 \\
\hline
\end{tabular}

Note-Values are the frequencies with which each of the three arms was selected as the second choice foltowing each first-choice error. "Shor delay" indicates that the interval between the end of the first choice and the start of the second choice was as short as the experimenter could manage (about 5-6 sec). "Long delay" indicates that the interval between the choices was at least $90 \mathrm{sec}$ in duration.

demonstrated a significant association between the location of the first-choice error and the location of the second choice $\left[\chi^{2}(4, n=88)=28.67, p<.05,\left[\chi^{2}(4, n=74)=\right.\right.$ $79.54, p<.05$, for short delay and long delay, respectively]. On long-delay trials, $82.4 \%$ of the animals' second choices were to the same location visited erroneously on the first choice. This level can be compared with the $75.6 \%$ accuracy observed for first choices. Both choices occurred after a 90 -sec delay. First choices were permitted $90 \mathrm{sec}$ following the forced choice sample runs, and second choices on the long-delay trials also occurred following a 90-sec delay. In contrast, on short-delay trials, only $61.4 \%$ of the animals' second choices were to the same location visited on the first choice, despite the shorter delay between the first and second choices.

\section{Discussion}

Unlike in previous experiments involving the secondchoice procedure, in this experiment all three choice alternatives continued to be available following a first-choice error. Despite a 90 -sec delay between the presentation of the sample and the presentation of the choice, first-choice accuracy was quite high in this experiment. Second-choice accuracy, however, was remarkably poor. The animals tended to repeat the choice that they had just erroneously made, even though they found no food in the incorrectly chosen arm. The rats remembered well the locations they had recently visited, but did not discriminate wisely between visits that required a return and those that should be avoided. We shall have more to say about this issue in the general discussion.

In another spatial memory experiment involving second choices without the removal of first-choice errors, pigeons were found to choose at above-chance accuracies on their first and their second choices (Dale, 1988). Pigeons were trained in a plus maze to find food during three forced choices. They were then returned to the center of the maze and given a free choice among the four arms. The correct response was to enter the arm that had not yet been entered. Second choices were permitted following first-choice errors, but the pigeon was not confined to the center of the maze and was allowed to make its second choice immediately following its first choice. Hence, perseveration of the first-choice error was not a possibility because the bird had to exit its first-choice arm in order to make a second choice.

The present experiment also demonstrated a difference in the distribution of second choices as a function of the delay interval between first and second choices. Unfortunately, duration of delay was confounded with the order in which these durations were tested. Short-delay trials were presented before all of the long-delay trials. As a result, we cannot know whether to attribute differences in performance following long and short delays to the duration of the delay or to the possibility that the rats eventually began to learn to avoid revisiting the locations of their first-choice errors. Experiment 2 was therefore conducted to disentangle these variables.

\section{EXPERIMENT 2}

Experiment 2 was conducted to substantiate the findings of Experiment 1 with naive animals and randomly presented delay conditions. A naive group of rats was trained to perform the spatial delayed matching-to-sample task and was then tested under conditions similar to those in Experiment 1.

\section{Method}

Subjects. The subjects were 4 male Sprague-Dawley rats bred by Simonsen Laboratory. They were approximately 60 days of age on arrival in the laboratory and were kept under the same conditions as the subjects in Experiment 1 . All rats were experimentally naive prior to the onset of training.

Apparatus. The apparatus used in this experiment was identical to that used in Experiment 1, except that it was located in a different experimental room. The room was well lit, with two doors on one side and a laboratory sink at one end.

Procedure. Upon arrival in the laboratory, the subjects were allowed to adapt for 5 weeks. During this period they were weighed daily and were provided with free food. Standard (free feeding) weights were taken to be the mean of the last three daily weights recorded prior to the onset of food deprivation. During the 6th week, the animals were gradually food deprived and exposed to the maze. Training began immediately upon the subjects' having reached the designated $80 \%$ of free-feeding weight. They received one 36-trial training session per day, and training continued until the rats achieved $90 \%$ correct performance in two successive sessions. Five additional sessions of overtraining were then given, followed by six test sessions similar to those in Experiment 1.

During training, there was no explicit delay between first and second choices, and only two arms were available for a second choice if an error was made on the first choice. The training intertrial interval was $30 \mathrm{sec}$.

The test procedure was identical to that of Experiment 1, except that the subjects received 36 trials per session instead of 24 , and the food cup was baited with only $1 / 2$ of a Froot Loop for the reward choice. Identical retention-interval durations were tested (short delay of 5-6 sec, long delay of $90 \mathrm{sec}$ ). On all trials, the delay between the sample and the first choice runs was $90 \mathrm{sec}$. Unlike in Experiment 1, in which all long-delay trials were run first, in this experiment the subjects received three sessions of each delay in ran- 


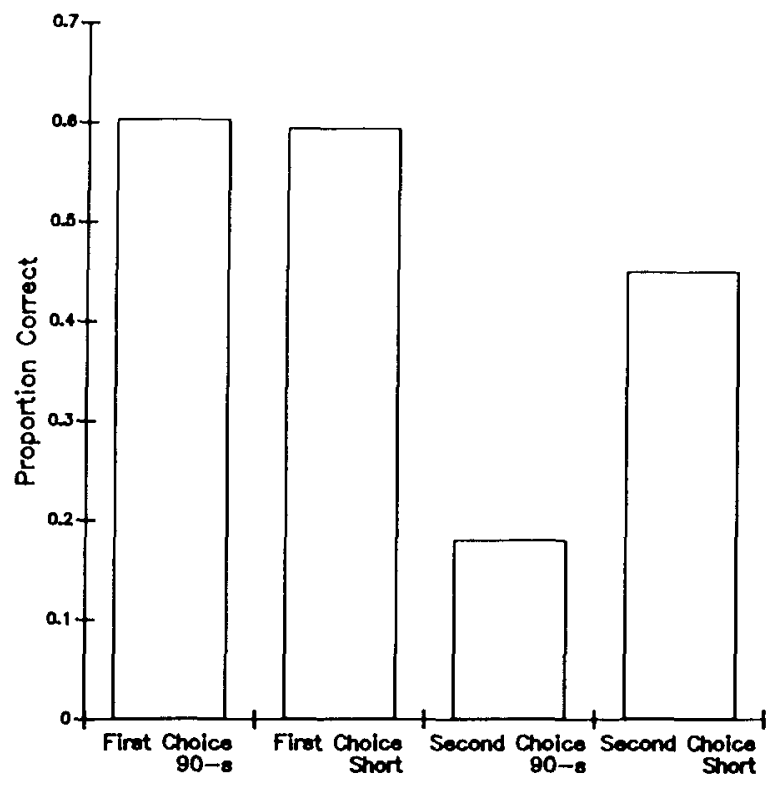

Figure 2. Mean first- and second-choice accuracy throughout Experiment 2. The first bar shows first-choice accuracy with a 90sec delay between first and second choices. The second bar shows first-choice accuracy with a 5-6-sec delay between choices. The next two bars show second-choice accuracy for short- and long-delay conditions, respectively.

dom order. In addition, long-delay intervals, both between the sample and the first choice and between the first and second choices, were spent in a plastic holding container instead of in home cages.

\section{Results}

Figure 2 shows the mean first- and second-choice accuracy averaged over all test sessions in Experiment 2. First-choice accuracy on short-delay trials $(59.5 \%)$ was not significantly different from that on long-delay trials $(60.4 \%)[t(2)=0.007, p>.05]$. Second-choice accuracy $(45.1 \%)$ did not differ from chance on trials with short delays between first and second choices $[t(3)=$ $2.39, p>.05$ ], and it was significantly below chance $(18.1 \%)$ with long delays $[t(3)=-5.38, p<.01]$.

The frequency with which each first choice followed each sample is shown in Table 4. As reported in Experiment 1 , first choices were distributed nonrandomly relative to the sample $\left[\chi^{2}(4, n=432)=141.24, p<.01\right.$, for short delays, and $\left[\chi^{2}(4, n=432)=146.56, p<.01\right.$, for long delays]. Again, locations of first-choice errors were found to be independent of the sample $\left[\chi^{2}(1\right.$, $n=175)=.56, p>.05$, and $\left[\chi^{2}(1, n=171)=1.56\right.$, $p>.05$, for short and long delays, respectively].

Table 5 shows the frequency with which each second choice followed each sample. Second-choice locations were found to be distributed nonrandomly relative to the sample for both short- and long-delay conditions $\left[\chi^{2}(4\right.$, $n=175)=13.69, p<.01$, and $\chi^{2}(4, n=171)=$ $20.52, p<.01]$. This is in contrast with the results of Experiment 1, in which the locations of second-choice errors were found to be independent of the sample location following short delays, but not following long delays. The direction of the relationship between samples and second choices, however, is different in the short- and long-delay conditions. An unusually large number of correct secondchoice responses occurred on short-delay trials when Arm $\mathrm{C}$ was the sample, compared with those occurring on comparable long-delay trials. In other respects, the distribution of second choices following first-choice errors is similar for both short and long delays.

Table 6 shows the frequency with which arms were selected as second choices following first-choice errors. Under both delay conditions, locations of first-choice errors and locations of second choices were found to be significantly related $\left[\chi^{2}(4, n=175)=107.77, p<.01\right.$, $\chi^{2}(4, n=171)=11.50, p<.05$, on trials with long and short delays, respectively]. On long-delay trials, $70.8 \%$ of second choices were to the same locations as firstchoice errors, whereas $40.0 \%$ were to the same locations on short-delay trials.

Table 7 shows the same data organized according to whether the second-choice response was correct, a perseveration of the first-choice error, or a response to the third arm. Choices were distributed differently between the short-delay and the long-delay trials $\left[\chi^{2}(2, n=346)=\right.$

Table 4

First Choices Following Each Sample in Experiment 2

\begin{tabular}{cccc}
\hline & \multicolumn{3}{c}{ Second Choice } \\
\cline { 2 - 4 } Sample & A & B & C \\
\hline \multicolumn{4}{c}{ Short Delay } \\
A & 106 & 22 & \\
B & 39 & 90 & 16 \\
C & 53 & 30 & 15 \\
& Long Delay & 61 \\
A & 109 & 16 & \\
B & 40 & 74 & 19 \\
C & 39 & 27 & 30 \\
\hline
\end{tabular}

Note-Values are the frequencies with which each of the three arms was selected following each sample. A total of 864 trials were presented.

Table 5

Second Choices Following Each Sample in Experiment 2

\begin{tabular}{cccc}
\hline & \multicolumn{3}{c}{ Second Choice } \\
\cline { 2 - 4 } Sample & A & B & C \\
\hline & Short Delay & \\
A & 9 & 15 & 14 \\
B & 17 & 22 & 15 \\
C & 17 & 18 & 48 \\
& Long Delay & & \\
A & 5 & 15 & 15 \\
B & 29 & 16 & 25 \\
C & 37 & 19 & 10 \\
\hline
\end{tabular}

Note-Values are the frequencies with which each of the three arms was selected as the second choice following each sample. Second choices were permitted only following first-choice errors. "Short delay" indicates that the interval between the end of the first choice and the start of the second choice was as short as the experimenter could manage (about 5-6 sec). "Long delay" indicates that the interval between the choices was at least $90 \mathrm{sec}$ in duration. 
Table 6

Second Choices Following Each First Choice in Experiment 2

\begin{tabular}{cccc}
\hline & \multicolumn{2}{c}{ Second Choice } \\
\cline { 2 - 4 } First Choice & A & B & C \\
\hline & Short Delay & & \\
A & 31 & 22 & 39 \\
B & 7 & 23 & 22 \\
C & 5 & 10 & 16 \\
& Long Delay & & \\
A & 58 & 15 & 6 \\
B & 10 & 26 & 7 \\
C & 3 & 9 & 37 \\
\hline
\end{tabular}

Note-Values are the frequencies with which each of the three arms was selected as the second choice following each first choice. "Short delay" indicates that the interval between the end of the first choice and the start of the second choice was as short as the experimenter could manage (about 5-6 sec). "Long delay" indicates that the interval between the choices was at least $90 \mathrm{sec}$ in duration.

Table 7

Second Choices Following Fach First Choice Error in Experiment 2

\begin{tabular}{cccc} 
& \multicolumn{3}{c}{ Second Choice } \\
\cline { 2 - 4 } & Correct & Perseveration & Other \\
\hline Short delay & 79 & 70 & 26 \\
Long delay & 31 & 121 & 19 \\
\hline
\end{tabular}

Note-Values are the frequencies with which the correct and each of the two kinds of erroroneous arms was selected as the second choice.

$35.61, p<.01]$. Correct responses were less likely and perseverations were more likely after long than after short delays.

\section{Discussion}

Experiment 2 replicated the main findings of Experiment 1 . Choice accuracy was somewhat lower in the second experiment than in the first, perhaps because of the briefer experience of the rats in Experiment 2. In both experiments, the rats generally preferred to return for the second choice to the arm they had visited during the erroneous first choice. As in the first experiment, this tendency to return to the location of the first-choice error was stronger when a long delay intervened between the first and second choices than when a short delay intervened. Unlike the subjects in the first experiment, the rats in Experiment 2 showed more evidence for memory of the sample during second choices on short-delay trials than they did following long delays, as can be seen in the stronger association between the location of the sample and the location of second choices, though this dependence did not necessarily result in more accurate choice performance.

A probable cause for the difference between the two experiments is the subjects' relative degrees of experience. The rats in Experiment 1 were highly overtrained on the spatial delayed matching-to-sample task, having served in several previous experiments. In contrast, the rats in Experiment 2 were only slightly overtrained, having con- tinued their training for only five sessions past a $90 \%$ acquisition criterion. As suggested earlier, this difference in experience is also likely to be the source of their lower first-choice accuracy.

As in the first experiment, repeats of first-choice error responses were more likely when a long delay intervened between the first and second choices than when a short delay intervened. This indicates that duration of delay was responsible for the increase in perseveration, and not the order in which the two delays were tested.

\section{GENERAL DISCUSSION}

The results of both experiments indicated that the second-choice distribution was more closely associated with the location of first-choice errors than with the location of the sample. However, in Experiment 2, it was found that in the short-delay condition second choices were also significantly related to the sample. Hence, the animals showed good evidence of being able to remember a visited arm, although they may have had problems discriminating between arms that should be revisited and arms that should be avoided.

It is surprising that the rats perseverated in choosing the first-choice arm even after they had the opportunity to discover that this choice was erroneous. It seems clear that the rats remembered well the arm they chose for the first choice, but they could not use the information that this memory was erroneous to avoid revisiting that arm. One source of perseverations might be that the rat made a first-choice error on the basis of an erroneous memory for the sample and then enhanced that memory by mistaking the first choice for a sample run and incrementing the strength of the representation for that arm. Hence, when the rat makes a second choice, its memory for the erroneously chosen arm is exceedingly high. The rat remembers well that it has visited the arm, but does not wisely distinguish between a "correct" and an "incorrect" memory.

In previous spatial delayed matching-to-sample experiments, access to the erroneous first-choice arm has been blocked before the rat is allowed a second choice, so perseveration of this choice has not been possible. Under these conditions, rats typically have shown above-chance second-choice accuracy. Furthermore, in previous experiments only a short delay has been used between first and second choices. Similar effects were obtained in the present experiments under comparable conditions. If repeats of the erroneous first choice are statistically removed, it is clear that the rats overwhelmingly preferred the sample arm over the remaining choice alternative when making their second choices. The ratios of sample to remaining alternatives were 30:4 and 13:00 in the first experiment and 79:26 and 31:19 in the second at short and long delays, respectively.

Another surprising finding is that the tendency to perseverate was stronger when a long delay followed the erroneous first choice than when a short delay followed. 
In interpreting the apparent anomalously good memory for the animal's first choice, one should keep in mind that the animal received the opportunity to make a second choice only when it failed to choose correctly on its first choice. By any of the memory models considered, the occurrence of a first-choice error indicates the presence of some relatively special memory conditions when compared with those present during correct first choices. The animal receives an opportunity to make a second choice only when its memory for the sample location is somehow impeded or made relatively less salient. At the time that the animal is selecting its first choice on these trials, its memory does not correspond exactly to the sample. The animal clearly has some information about the location of the correct sample arm (as shown, for example, in above-chance preference for the sample relative to the remaining alternative), but this memory does not directly control its response. The state of the animal's memory is clearly different from that on trials in which its first choice corresponds to the sample. Put simply, the rat has an erroneous representation of its sample experience when making its first choice on trials on which it makes an error. This erroneous representation could occur through faulty encoding of the sample (e.g., the rat could be mistaken about the location of the arm on which it was fed), or it could have resulted from changes that occurred during the retention interval. We currently have no way to determine the state of the animal's memory in the interval between the presentation of the sample and the occurrence of the first choice, but it seems most likely to us that the erroneous memory seen on first-choice errors is the result of an erroneous encoding of the sample. The perseveration of the first-choice error response when second choices are made suggests that the first-choice error is not simply the result of a momentary fluctuation in the rat's memory, but, rather, the result of a "hard" unrecoverable error in the memory representation. The animal makes its first choice on the basis of a faulty representation of the sample information and then persists in making its second choice on the basis of exactly the same information. It is prevented from this perseveration in the standard version of the task by closing off the erroneously chosen arm. Removing the erroneously coded arm from the available alternatives allows the animal to "uncover" the information it has about the correct identity of the sample and allows it to choose with above-chance accuracy (compare the distribution of choices of the sample arm with the remaining arm when perseverations are statistically removed). In contrast, when the erroneously chosen arm is not removed from the set of available choices, then the rat continues to make its response on the basis of the same memory that it used to guide its first choice. As a result, the animal perseverates.

The stronger perseveration of first-choice errors after long than after short retention intervals between the first and second choices also substantiates the interpretation of first-choice errors as the result of inappropriate encoding. The interval between the sample and the first choice was the same as the interval between the first choice and the second choice. The occurrence of the first choice provided an indication of the state of the animal's memory at that point. Perseveration of the same response $90 \mathrm{sec}$ later indicates that the same memory persisted over a similar 90-sec interval. At the same time, the higher second-choice accuracy following a short retention interval indicates that some information about the identity of the sample was still present for a time and still affected choices. Hence these results support the hypothesis that memory coding in this task is not simply an all-or-none process, but instead is the result of gradually changing representations.

Perseveration of the first-choice error was higher when long delays intervened between the two choices than when shorter delays intervened. This result might be explained by noting that as the delay between the first choice and the second choice increased, so did the delay between the sample and the second choice. Hence, perseverations might have increased simply because the partial memory for the sample had decayed even more by the time the second choice was made following long delays, and so it was less influential. Simulations have demonstrated that such an explanation can account for the rats' performance provided that the rate of memory loss for the sample information is faster than the rate of loss of the memory leading to the incorrect first choice.

For example, according to one model (the relative strength model), the rat maintains a memory element for each of the three alternatives. Each of these memory elements is a random variable representing the strength of the memory for the corresponding alternative. Ordinarily, the exposure to the sample causes the corresponding memory element to increase in strength, and the delay interval causes it to decrease. On the average, the strength of the element corresponding to the sample is therefore likely to be stronger than the strength of the other two elements; but on any given trial, one of the other elements might by chance be stronger, and an error will result. According to this model, the probability of making a specific choice depends on the strength of the corresponding memory element, relative to the sum of the strengths of the three elements. The probability of a specific choice, therefore, can increase if its strength remains relatively unchanged but the strengths of the other two elements decrease. A concrete example may help to illustrate the point.

Table 7 shows the frequency of second-choice responses that were correct, perseverations of the first choice, or were choices of the remaining arm. The corresponding proportions were $0.45,0.40$, and 0.15 for the short-delay condition and $0.18,0.71$, and 0.11 for the long-delay condition. Many different combinations of memory strengths for the three elements could yield these choice proportions. All that is required is that the ratio of the three memory elements to their sum $\left[X_{i} /\left(X_{1}+X_{2}+X_{3}\right)\right]$ be in about the same proportion as the ratio of responses to their sum. For example, at the time of the short delay, the memory 
strengths for the three elements of $0.70,0.64$, and 0.23 match closely the corresponding choice proportions [0.45 = $0.70 /(0.70+0.64+0.23), \quad 0.40=0.64 /(0.70+0.64$ $+0.23)$, and $0.15=0.23 /(0.70+0.64+0.23)]$. The corresponding values after a long delay might be $0.031,0.120$, and 0.019 . These strengths match closely the observed proportion of second-choice responses following a long delay. Other combinations of putative memory strengths also yield matching response proportions, but all sets share the property of requiring slower loss of strength for the perseverated choice than for the other alternatives.

The preceding model treats each memory element as independent of the other two. In the matching-to-sample task, however, exactly one of the alternatives is always correct on every trial. Therefore, the alternatives are not independent of one another. To the extent that the animal can rule out one of the alternatives as the correct choice, the animal can increase its confidence that one of the others is the correct choice (see Roitblat, Penner, \& Nachtigall, 1990). The drift model (Edhouse \& White, 1988; Roitblat, 1984b; Roitblat \& Harley, 1988) explicitly includes this interdependency among representations by conceiving of the animal's memory as a pointer located in a two-dimensional, rather than a three-dimensional space. As the pointer moves farther from one of the alternatives, it necessarily moves closer to at least one of the others. Figure 3 shows the average locations in this memory space that would be occupied by the pointer following short delays and following long delays in order to produce the choice proportions observed in Experiment 2. The top corner of the space represents the location of the correct choice, the lower left-hand corner of the space represents the position of the alternative chosen as the perseverated first-choice error, and the third corner of the space represents the position of the remaining alternative. The pointer's position is assumed to vary randomly from the specified average locations over trials, and the animal is assumed to choose the alternative to whose representation the pointer is closest at the time of choice.

According to the drift model, an erroneous first choice occurs because the pointer is closer to the erroneous alternative than to the correct alternative at the time the choice is made. Perseveration occurs when the average location of the pointer is still relatively closer to the incorrect choice after a delay. When errors occur, the location of the pointer is likely to be nearly the same distance from the correct alternative and from one of the incorrect alternatives. The occurrence of the error implies that the position of the pointer is at least slightly closer to the incorrect than to the correct alternative. Small perturbations in the position of the pointer thus are likely to result in different choices' being made. After a short delay, the pointer can easily drift from a position that is slightly closer to the incorrect alternative (the cause of
Drift Model Memory Space

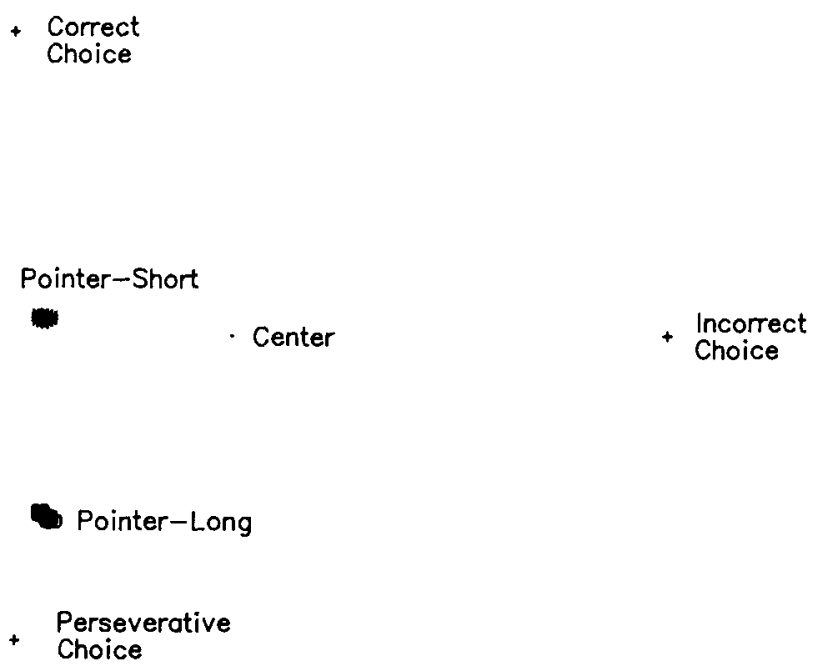

Figure 3. The memory space for the drift model representation of second choices in Experiment 2. Pointer-Short shows the approximate location of the memory pointer after a short delay. PointerLong shows the corresponding location of the pointer after a long delay. The Center of the space is equidistant from the representations of each of the alternatives at the vertices of an equilateral triangle. Choice proportions correspond approximately to the average position of the pointer at the designated time.

the erroneous first choice) to a position that is slightly closer to the sample (which would cause a correct second choice).

In order for perseverations to be more likely after long than after short delays, the pointer would have to be even more likely to be in a position relatively near the erroneously chosen alternative after a longer delay. This implies that the pointer actually moves toward the alternative that controlled the erroneous first choice. As a result, it is substantially closer to the perseverated alternative after a long delay than after a short delay. The model thus suggests some kind of rehearsal process. In fact, both models seem to imply a rehearsal process that either selectively strengthens or selectively maintains the alternative corresponding to the first choice.

Rehearsal has frequently been suggested as an important mechanism in animal memory (Grant, 1984; Maki, 1981 ; cf. Wright, 1989). Roitblat (1984b), in the original description of the drift model, argued that the complete memory system consists of an initial encoding process that identifies the sample stimulus and drives the pointer for a time after the sample is removed. It is conceivable that the rat's excellent ability to maintain spatial information over long periods of time is mediated at least in part by some kind of rehearsal process that maintains the identification in the encoding process for extended periods of time and continues to drive the pointer (or continues to 
allow maintenance of the representation). In this view, the animal continues to strengthen over the delay the representation of the remembered sample (if second choices are involved, its memory is not for the correct sample, but most strongly for the item to which the first-choice error is directed).

A possible alternative explanation, as suggested above, is that the animal mistakes its first-choice run for a sample run. When the animal makes its second choice among the three alternatives, it somehow mistakenly behaves as if it were making its first choice and bases this choice on its memory of the first-choice run rather than on a memory of the preceding sample run. It then returns to the most recently visited location, as it does on its first choice. This explanation by itself is implausible. If the rat strengthened its representation of the erroneous first choice immediately following this choice, it should show high rates of intrusions on the immediately following short-delay second choice. Instead, intrusions were more likely following long than following short delays, indicating that the relative strength of the representation of the incorrectly chosen alternative must grow slowly and continuously during the delay rather than quickly at the start of the interval.

In addition, there are substantial differences between the sample run and the first-choice run. Only one arm is accessible on the sample run, food is available, it can be seen remaining in the arm, and so forth. In contrast, during the first-choice run, all three doors are open, no food is present in the chosen arm, and so forth. These differences were exaggerated during Experiment 2, because the rats spent the intertrial intervals in their home cages but spent the delay periods in a highly salient, white plastic bucket.

Furthermore, Herrmann, Bahr, Bremner, and Ellen (1982) found that rats performing a win-stay version of Maier's three-table problem (Maier, 1932) never performed above chance in returning to a location that did not contain food. Similarly, in another spatial task (Olton, Walker, Gage, \& Johnson, 1977), rats avoided locations that they had visited and had found devoid of food. In the latter experiment, the food was located on the top of one of three randomly selected towers. A hungry rat was released into the enclosure and allowed to visit the towers. On each visit, the probability of visiting the tower containing the food was equal to chance, but the probability of revisiting a tower that had been found empty was near zero (see also DiMattia \& Kesner, 1984). Both of these tasks demonstrate that rats are ordinarily quite good at remembering and avoiding locations without food. These tasks differ from the present one, in which the animals were required and explicitly trained to return to the arm that had been baited for the sample run. Within a session, the same arm served repeatedly as either the baited sample arm or as a distracting alternative. Perhaps this difference led to some kind of confusion. Subsequent experi- ments will be necessary for the investigation of these issues.

The results of the present experiments also suggest that encoding and maintenance of the rat's spatial memory may be a more complex process than has previously been considered. They suggest that current models may be too simplistic to account for the rat's performance. Current models tend to emphasize relatively passive processes such as recognition and maintenance, whereas the present data begin to suggest more active kinds of processing such as rehearsal. Procedures such as those used in the present study suggest ways to investigate these processes in animal memory that may have important implications for understanding both the cognitive/behavioral aspects of animal memory and its neurophysiological substrate.

\section{REFERENCES}

DALE, R. H. I. (1988). Spatial memory in pigeons on a four-arm radial maze. Canadian Jourmal of Psychology, 42, 78-83.

DiMattia, B. V., Kesner, R. P. (1984). Serial position curves in rats: Automatic versus effortful information processing. Journal of Experimental Psychology: Animal Behavior Processes, 10, 557-563.

EDHOUSE, W. V., WhITE, K. G. (1988). Sources of proactive interference in animal memory. Journal of Experimental Psychology: Animal Behavior Processes, 14, 56-70.

GraNT, D. S. (1984). Rehearsal in pigeon short-term memory. In H. L. Roitblat, T. G. Bever, \& H. S. Terrace (Eds.), Animal cognition (pp. 99-116). Hillsdale, NJ: Erlbaum.

Grant, D. S., \& RoberTS, W. A. (1973). Trace interaction in pigeon short-term memory. Joumal of Experimental Psychology, 101, $2 \mathrm{l}-29$.

Herrmann, T., Bahr, E., Bremner, B., Ellen, P. (1982). Problem solving in the rat: Stay versus shift solutions on the three-table task. Animal Learning \& Behavior, 10, 39-45.

MAIER, N. R. F. (1932). A study of orientation in the rat. Journal of Comparative Psychology, 14, 387-399.

MAKI, W. S. (1981). Directed forgetting in pigeons. In N. E. Spear \& R. R. Miller (Eds.), Information processing in animals (pp. 199. 226). Hillsdale, NJ: Erlbaum.

Olton, D. S., Walker, J. A., Gage, F. H., III, Johnson, C. T. (1977). Choice behavior of rats searching for food. Learning \& Motivation, 8, 315:331.

RoberTs, W. A., \& Grant, D. S. (1976). Studies in short-term memory in pigeons using delayed matching to sample procedure. In D. L. Medin, W. A. Roberts, \& R. T. Davis (Eds.), Processes of animal memory (pp. 79-112). Hillsdale, NJ: Erlbaum.

RoItblat, H. L. (1980). Codes and coding processes in pigeon shortterm memory. Animal Learning \& Behavior, 8, 341-351.

RoItBLAT, H. L. (1982). The meaning of representation in animal memory. Behavioral \& Brain Sciences, 5, 353-406.

Roitblat, H. L. (1984a). Pigeon working memory: Models for delayed matching-to-sample performance. In $\mathbf{M}$. L. Commons, A. R. Wagner, \& R. J. Herrnstein (Eds.), Quantitative analyses of behavior: Discrimination processes (Vol. 4, pp. 161-181). Cambridge, MA: Ballinger.

RoITBLAT, H. L. (1984b). Representations in pigeon working memory. In H. L. Roitblat, T. G. Bever, \& H. S. Terrace (Eds.), Animal cognition (pp. 79-97). Hillsdale, NJ: Erlbaum.

RoItblat, H. L. (1987). Introduction to comparative cognition. New York: W. H. Freeman.

Rortblat, H. L., \& Harley, H. E. (1988). Rat spatial delayed matching-to-sample performance: Acquisition and retention. Jourmal of Experimental Psychology: Animal Behavior Processes, 14, 71-82.

Roitblat, H. L., Harley, H. E., Helweg, D. A. (1989). The 
effects of scopolamine on proactive interference and spatial delayed matching-to-sample performance in rats. Psychobiology, 17, 402-408.

Roitblat, H. L., Penner, R. H., \& Nachtigall, P. E. (1990). Matching-to-sample by an echolocating dolphin (Tursiops truncatus). Journal of Experimental Psychology: Animal Behavior Processes, 16, 85-95.

Roitblat, H. L., Scopatz, R. A. (1983). Sequential effects in delayed matching-to-sample. Joumal of Experimental Psychology: Animal Behavior Processes, 9, 202-221.

WriGHT, A. A. (1989). Memory processing by pigeons, monkeys, and people. In G. H. Bower (Ed.), The psychology of learning and motivation (Vol. 24, pp. 25-70). San Diego, CA: Academic Press.

\section{NOTES}

1. Analysis of the distribution of first-choice errors presents some special problems, because the main diagonal of this table consists of structural zeroes-these values are zero by definition. Selection of the choice arm that matches the sample is a correct response, and hence, correct choices cannot be included in the analysis of first-choice errors. Only the remaining entries in each row are used to calculate the expectation.

(Manuscript received June 17, 1989; revision accepted for publication April 28, 1992.) 